Témoigner Témoigner. Entre histoire et mémoire

Getuigen Revue pluridisciplinaire de la Fondation Auschwitz

$125 \mid 2017$

Histoire et mémoire de la persécution des

homosexuel-le's par les nazis

\title{
Présentation. Histoire et mémoire de la persécution des homosexuel-le-s par les nazis
}

Inleiding. De vervolging van homoseksuelen door de nazi's

\section{Régis Schlagdenhauffen}

\section{(2) OpenEdition}

Journals

Édition électronique

URL : https://journals.openedition.org/temoigner/6319

DOI : $10.4000 /$ temoigner.6319

ISSN : 2506-6390

Traduction(s) :

Inleiding. De vervolging van homoseksuelen door de nazi's - URL : https://journals.openedition.org/ temoigner/6339 [nl]

Éditeur :

Éditions du Centre d'études et de documentation Mémoire d'Auschwitz, Éditions Kimé

Édition imprimée

Date de publication : 1 octobre 2017

Pagination : 60-71

ISBN : 978-2-930953-01-4

ISSN : 2031-4183

Référence électronique

Régis Schlagdenhauffen, « Présentation. Histoire et mémoire de la persécution des homosexuel-le-s par les nazis », Témoigner. Entre histoire et mémoire [En ligne], 125 | 2017, mis en ligne le 24 décembre 2021, consulté le 03 février 2022. URL : http://journals.openedition.org/temoigner/6319 ; DOI : https:// doi.org/10.4000/temoigner.6319 


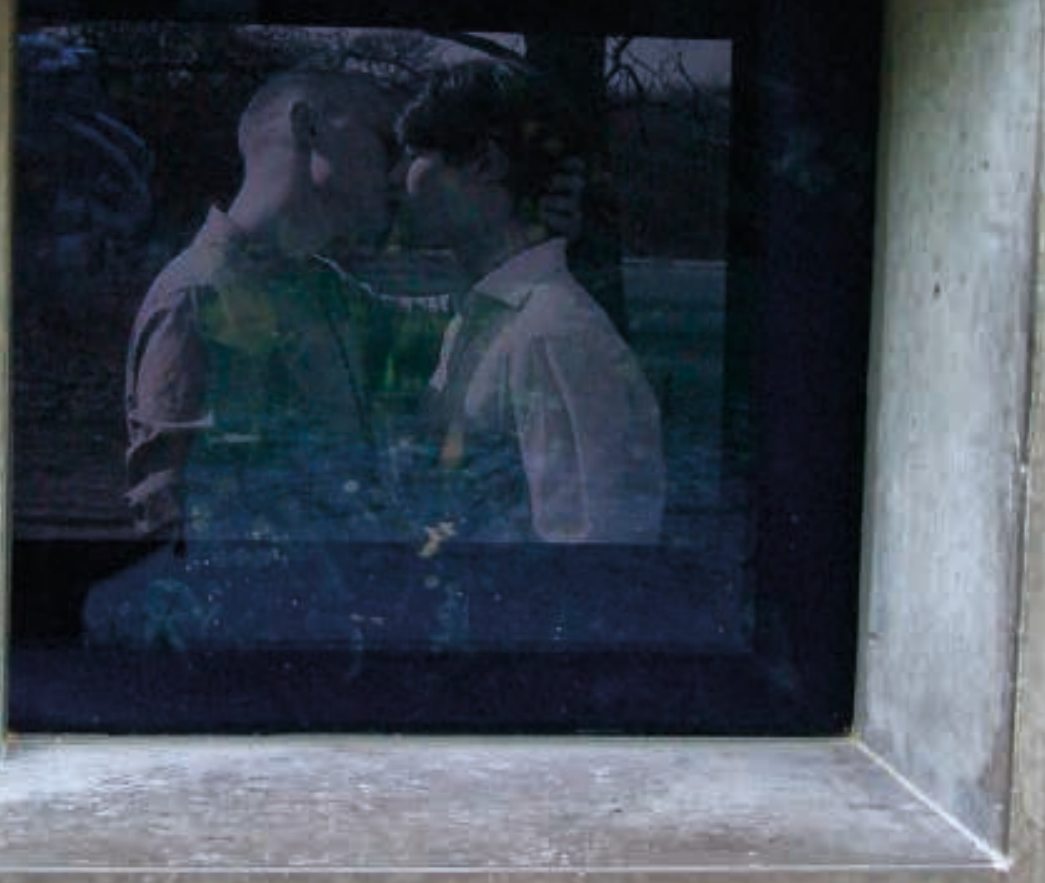

\section{HISTOIRE ET MEMORREDELA PERSECUTION DES HOMOSEXUEL-LE-S PAR LES NAZIS}

La connaissance historique de la persécution nazie des homosexuel-le-s et de leur déportation a connu des avancées significatives ces dernières années du fait de la multiplication des recherches sur le sujet.

Dans le cadre de ce dossier, la parole est donnée à des chercheuses et chercheurs reconnus ainsi qu'à de jeunes doctorants et docteurs. Les éclairages qu'ils apportent concernent aussi bien la question de la destinée singulière des femmes et des hommes homosexuels durant la Seconde Guerre mondiale, que la manière dont la mémoire des victimes homosexuelles du nazisme a évolué depuis la fin de cette guerre.

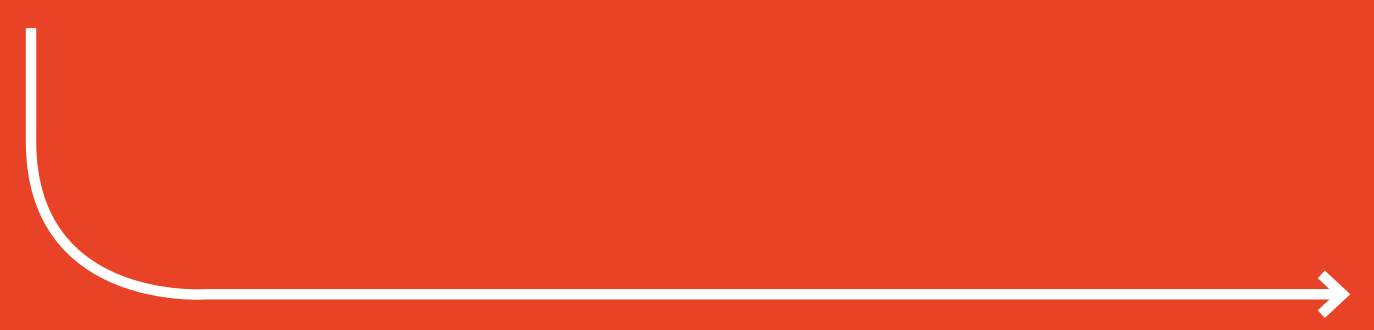


gories de la société, certains États ont fait le choix de s'engager pour la défense deslibertés indiviolles, tout du moin celles relatives à lorientation sexuelle.

Force est de constater qu'un long chemin a été parcouru du point de vue de la documentation relative aux persécutions endurées par les homosexuels comme l'attestent les quatre articles qui constituent ce dossier. Le premie signé de la plume de Florence Tamagne, nous rappelle que la persécution nazie des homosexuels d'Allemagne s'est déroulée sur une période de douze an (1933-1945). Conduisant à plus de 100000 arrestations à travers tout le pays et au déploiement de formes de persécution jusqu’alors quasi inédites, elles témoignent du souci qu’avait le système national-socialiste d'éradique l'homosexualité au moyen du déploiement combiné de dispositifs coercitifs et prophylactiques : traitement médicaux infligés sous la contrainte, castration, expérimentations de toute sortes. Ces dispositifs ont conduit à briser des dizaines de milliers de vies de ces femmes et hommes envoyés en prison et en camps de concentration, dont seul quelques-uns ont survécu.

À sa suite, Claudia Schoppmann, propose une synthèse des recherche sur les femmes lesbiennes sous la botte nazie. S’appuyant sur des témoignages de survivantes de la déportation, Schoppmann montre la vivacité d'un sous-culture lesbienne à l'intérieur de l'univers concentrationnaire, mais auss les tensions inhérentes à un univers fermé et monosexué qui, pour différentes raisons, avait tendance à mettre surlatouche lesfemmeshomosexuelles. Cet article nous offre ainsi une plongée dans un univers qui n'est pas exempt de violences, notamment sexuelles.
S’agissant de l'article de Jean-Luc Schwab, l'auteur y décrit les manière dont la répression de l'homosexualité a pu s'exercer en France durant la Seconde Guerre mondiale. Selon qu'il s'agisse de la France dite libre, de la France occupée ou encore de la France annexée (Alsace-Moselle), trois forme de persécution judiciaire et extra-judiciaire ont pu être démontrées. Dans le premier cas, l'Etat a fait appel, dès 1940, à l'internement administratif. En zone occupée, tout Français qui entretenai une relation intime avec un soldat d'occupation était susceptible d'être poursuivi par une cour spéciale alle mande au titre de l'article 175 du Code pénal du « $\mathrm{III}^{\mathrm{e}}$ Reich» réprimant les relations «contre nature». Enfin, en France annexée, le régime nazi a ag en combinant persécution extra-judiciaire et judiciaire dans le cadre de la Gleichschaltung, la mise au pas, de ces trois départements del'Est de la France. Au final, pas moins de 370 homosexuel ont été victimes de cette politique et plupart de ces hommes ont eté internés pour ce motif dans un camp de rééducation spécial (Schirmeck).

Enfin, Sam Seydieh, qui a récemment soutenu sa thèse de doctorat sociologie, s'interesse dans son article à la façon dont les differenten forme d'évocation de la déportation pour motif d'homosexualité (témoignages, recherche des traces, commemorations) contribuent à creer un registre d'engagement singulier au sein de l'espace militant LGBT (lesbien, gay, bi et trans). Il montre ainsi que l'espace militant, a cours de ses transformations et malgré ses divergences, constitue un lieu d'interaction entre plusieurs niveaux de mémoire (mémoires empruntees de militant.e.s, ménoire historique élaborée par les militant.e.s autour d'un passé commun, mémoires communes des luttes). Son article permet de comprendre le rôle de la socialisation militante dans la transmission du sens de ce passé de persécution. Pour expliquer les ressorts d’un engagement basé sur a reference a la déportation pour moti d'homosexualité, son article saisit ces engagements par le biais d'une analyse descontextes institutionnels dunepart et des carrières militantes d'autre part.»

\section{État de la recherche}

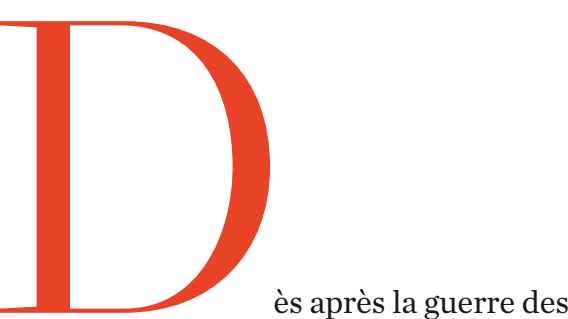
militants de la és après la guerre des int et reportages sur les souffrances endurées par les homosexuels du fait de la répression nazie faconnant ainsi les répression nazie façonnant ainsi les revue néerlandophone Levensrecht, éditée à Amsterdophone Levenssrecht, edilance. Malgré les imprécisions dont ils font preuve et le ton parfois militant qui ctérve le ton parfoismilitant qui ils constituent néanmoins le socle des travaux historiques qui suivront ${ }^{1}$ C'est travaux historiques qui suivront ${ }^{1}$. Cest ensuite en Allemagne que des articles sur le sujet seront publies dans des revues associatives homosexuelles
(1) Cf. Régis Schlagdenhauffen, «Le Dodenherdenking à Témoigner. Entre Histoire et Mémoire,
$n^{\circ} 110,2011$, p. 114-126. 
durant les années 1950, permettant l'émergence, certes timide, de premier travauxà partir des années 1960. Témoi d'une époque conservatrice, louvrage
Das Große Tabu, le grand tabou (1967) Das Große Tabu, le grand tabou (1967), publié par le sexologue allemand Wilhart Schlegel, cherchait en premier lieu à rendre publique la question del'homophobie et notamment celle qui s'étai manifestée d'une manière encore inédite à travers la persécution nazie de homosexuels. Puis, grâce à «mai 1968», moment séminal qui a permis la const tution de groupes et d'association d'émancipation gays et lesbiennes dan plusieurs pays d'Europe, nous pouvon observer l'emergence d'une volonté de savoir. Comprendre le calvaire enduré par leurs ainés participe pour ces militantes et militants de la memoire d'u désir plus global de conjurer l'oubli de «l'histoire officielle». Dans cette entre prise, les prises de position sur le sujet se multiplient à partir des annees 1970 période où des militantes et militant se reapproprient le triangle rose por par les déportés homosexuels dans les camps. La publication des mémoires de Heinz Heger, premier temoignage d'un déporté homosexuel, n’y est pas étrangère puisquelle constitue la première possibilité d'identification des militants des mouvements de libération gay avec une trajectoire de victime. Les hommes au triangle rose, «best-seller» publié en 1972 en allemand retrace en effet la trajectoire d déporté homosexuel autrichien Heinz Heger. Lui fera suite la publicatio d'une étude sociologique et historique intitulée Société et homosexualité. A moyen d'une étude quantitative, Rüdiger Lautmann y montre quen raison de differents déterminants sociaux les homosexuels avaient une espérance de viebien moindre que lesinternés «hétérosexuels» en camp de concentratio
(Lautmann, 1977, p. 300-65). Ensuite, es travaux sur le sujet se multiplient. Notons à cet endroit deux thèses de doctorat. Tout dabord celle de Burkhard Jellonek, Les homosexuels sous la croix gammée (1990), qui compare à parti des archives de la Gestapo les modalités de la persécution dans une grande métropole allemande (Düsseldorf), une ville moyenne (Würzburg) et une région rurale, le Palatinat. Ensuite, la these de Claudia Schoppmann (1997) qui, aujourd'hui encore, est considéree comme la réference concernant les femmes lesbiennes sous le «III Reich» permet d'aborder pour la première foi la question sous l'angle du genre. Ce travaux ouvrent la voie à différent. ouvrages exclusivement dédiés à l'histoire des homosexuels d'Allemagne durantle «III Reich» dont ceux de Thomas Rahe (1999) et d'Olaf Mussmann (2000). Parallèlement, les première études etrangères sur les homosexuel dans les territoires occupés et annexés par les nazis se multiplient, elles aussi, impulsées par un groupe de chercheurs néerlandais (Koenders, 1996).

Enfin, en 2004, l'historien Günter Grau édite un ouvrage qui deviendra lui aussi une référence, car retraçant au moyen darchives administratives, la mise en œuvre progressive de la persécution nazie des homosexuels en Allemagne et dans certains territoires annexés.

À côté de la littérature scientifique existent aussi des témoignages d'homosexuels persécutés durant le $111{ }^{\circ}$ Reich et plus généralement la Seconde Guerre mondiale. Certains sont de nature autobiographique - comme celui de l'Autrichien Heinz Heger précédemmentévoque (1972) - ou ceux de Pierre Seel (publié avec le concours du journaliste Jean Le Bitoux en 1994) et de Rudolph Brazda qui, en juin 2008 à lâge de 95 ans, a rendu publique sa qualité de déporté homosexuel. JeanLuc Schwab s'est chargé de l'écriture de sa biographie qui a donné lieu à sa publication en français en 2010. Cette dernière s'inscrit à la suite de recueils de témoignages consignés et édités par des collègues allemands. Citon à cet endroit le livre de Lutz van Dijk, publié en 1992, qui présente onze trajectoires d'homosexuels ayant survéc à la guerre ainsi que la série Lebensegeschichten, histoires de vie, coordonnée par Andreas Sternweiler, qui retrace la vie de quatre homosexuels allemands le photographe Albrecht Becker, l'éclaireur scout Heinz Dörmer, le professeu de litterature Richard Plant et l'historien de l'art Christian Adolf Isermeyer.

Enfin, un tel tour d'horizon ne saurait être complet sans mentionne la production theatrale, cinematographique et littéraire ni les dernières publications francophones. Écrite pa Martin Sherman et jouée pour la première en 1979 à Londres, puis en 198 à New York et enfin en 1981 à Paris Bent est la pièce de théâtre qui met en scène la persécution des homosexuel par les nazis². Elle a été suivie par le documentaire cinématographique Paragraph 175, réalisé par Rob Epstein et Jeffrey Friedman en $1999^{3}$. Plus prè de nous, deux œuvres litteraires on été publiees l'an dernier en français. La première intitulée Les guérir (Olivier Charneux) relate l'experience du médecin danois Carl Vaernet qui réalisa des expériences médicales sur des groupes d'homosexuels au camp de Buchenwald. La seconde, Je suis en vie et tu ne mentends pas de Daniel Arsand nous fait vivre le retour de Déportation d'un certain Klaus, dans un monde où ersonne ne l'attendait plus. Du côté des travaux historiques, les publications francophones ne sont pas en reste témoignant de l'interét contemporain que suscite la question de la «Déportation homosexuelle» comme le confirment les ouvrages suivants, tous publiés au cours des annees 2010 : Le III Reich et les homosexuels de Thomas Rozec (2011) ainsi que les études historiques de Mickaêl Bertrand (2011), et de Régis Schlagdenhauffen $(2011,2017)$

Les principaux acquis de ces travaux dont la suite de cet article se propose doffrir une synthèse permettan de mettre en lumière la complexité de ce que furent les persécutions des homosexuels durant la Seconde Guerre mondiale, mais aussi les zones de liberté et la mise en œuvre de législation progressiste durant la Seconde Guerre mondiale dans quelques pays d'Europe.

\section{Persécutions}

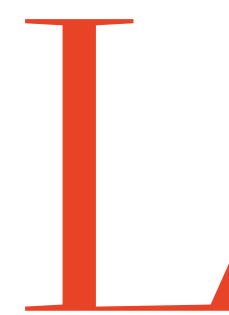

a persécution nazie a affecté de maniere differentielle les homosexuels selon leur genre et leur nationalite. D'une maniere generale, (a) des hommes que des femmes et qu'il a History: Toward a Gay Holocaus Litterature, The German
Quaterly, no 70 (1), 1997, p. 18-26. (3) Rob Epstein et Jeffrey Friedman, Paragraph 175, USA 
pourchassé les homosexuels d'Allemagne et des territoires annexés tou en ne se préoccupant que très margnalement de cette question dans les territoires occupés. Ceci nous oblige reconnaître que tous les homosexuels d'Europe n’ont pas été déportés. L'histoire et les modalités de cette persécution en Allemagne étant exposées dan les articles de Florence Tamagne et de Claudia Schoppmann, la suite du propos concernera deux États sous domination nazie: l'Autriche et les Pays-Bas témoignant des facultés d’adaptation d système nazi.

Suite à l'Anschluss, l'Autriche e intégrée au «III ${ }^{e}$ Reich» en 1938 et droit autrichien y reste en vigueur. A différence de l'Allemagne, les relation homosexuelles y sont condamnées, quel que soit le sexe des partenaire en vertu des articles 129 et 130 du Code pénal autrichien. Pour cette rason, l'Autriche comporte le plus gran nombre de victimes lesbiennes d nazisme parmi les Etats annexes au Reich. Selon Angela Mayer, des dizaine defemmeslesbiennes ont effectivemen été arrêtées et condamnees. La plupart de celles persécutées par les nazis on soit éte détenues en camp de concentration, soit en camp de rééducatio par le travail (Arbeitserziehungslager) ou encore internees en institution psychiatriques. ${ }^{4}$ Sagissant plus précsément de la ville de Vienne, 79 femmes ont fait l'objet d'une condamnation en vertu du $\$ 129$ entre 1938 et 1943 versus 1400 hommes durant la même période

Aux Pays-Bas, malgré l'absence de pénalisation des relations homosexuelles entre adultes consentants, police neerlandaise avait pris ' habitud de consigner lidentite des personn ayant des pratiques homosexuelles dan des fichiers spéciaux depuis $1920^{6}$. Pieter Koenders, auteur d'une étude sur a repression de l'homosexualité aux ays-Bas occupés, montre qua partir du 31 juillet 1940, une ordonnance nazie es promulguée par l'armée d'occupation allemande rendant les relations homosexuelles entre adultes consentant condamnables d'une peine de cinq an. sur le modèle de l'article 175 du Code pénal allemand. Elle complète l'article 248bis du Code pénal néerlandais qu condamnait les relations homosexuelles entre majeur et mineur. Cependant parce que cette ordonnance emanait de l'occupant, la police néerlandaise ne s'est pas rendue particulièrement coopérative quant a son application. Ce constat a conduit loccupant à changer de stratégie et à créer un bureau central d'enregistrement des homosexuels à La Haye. De plus, afin d'optimiser la communication avec la police du Reich, le système de codification a été harmonisé avec celui en vigueur en Allemagne ${ }^{7}$. A partir de 1943, une Centrale pour la lutte contre l'homosexualite apparaît au sein de lorganigramme de la police des mouts néerlandaise. Dès lors, nou pouvons affirmer que quatre objectifs de loccupant sont ainsi réalisés: la disparition des associations homosexuelles, la condamnation penale de l'homosexualité (masculine), l'enregistrement de personnes qualifiees d homosexuelles et enfin la création de brigades de police spécialisées dans la lutte contre 'homosexualité. Finalement, s'agissan de la repression des personnes homosexuelles aux Pays-Bas, nous arrivon aux chiffres suivants: 138 comparution. en justice en vertu de l'ordonnance $81 / 40$ entre 1940 et 1943,90 condamnations et 10 internements en hôpital psychiatrique. Parallèlement, 164 homme ontcomparuenjusticepour violationde l'article 248bis et 77 ont été condamnés à une peine d'emprisonnement (Grau, 2004, p. 270). Enfin, Koenders relève le cas de personnes non condamnées par la loi, mais tout de mème persécutées e raison de leur homosexualité, ces personnes sont toutes juives. Il en va ains de trois hommes et d'une femme arrêtés en 1942 puis déportés et assassinés dan e cadre de la «Solution finale» (Koenders, 1996).

\section{Les homosexuels \\ dans les camps}

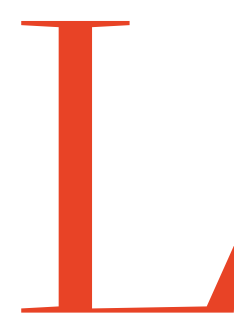

es données relatives aux es révèlent qu'environ 50000 homme ont été condamnés par la justice pénale nazie à des peines de prison, de maison de redressement ou encore aux camp de travaux forcés. Entre 5000 et 1000 hommes ont quant à eux été internés en camp de concentration pour homosexualite. Dans les camps, les homosexuelsformentcependant une minorit tatistique puisquils constituent moin de $1 \%$ des internés. Isoles géographiquement et socialement deleurscamarades, ils sont a la fois victimes des SS et de leurs compagnons d'infortune. L'homophobie anbiantedanslescanips senb être le reflet de celle existant dans l société. Effet du «pouvoir absolu», es homosexuels sont prioritairement désignés par leurs camarades «hétérosexuels » pour être transférés vers les commandos et camps aux conditions de travail et de vie les plus rudes. Leur étiquette d'homosexuel les prédestine aussi à servir de cobayes (émasculations à Sachsenhausen, «épreuves de normalisation » à Ravenbrück, «traitement hormonal» à Buchenwald). Comparée aux autres groupes de détenus allemands, lesperance de vie des homosexuels est nettement inférieure (tout comme leur probabilite deetre un jour libéré). Qui plus est, parmi les homosexuels qui sont libereses, un nombre non négligeable dentre eux sont incorpores de force dans la Wehrmacht ou dans des bataillons spéciaux de la SS (surtout après 1942). Quelques cas exceptionnels d'homosexuels ayant su s'élever dans la hiérarchie des camps nous sont toutefois parvenus. Ces derniers semblent se comporter a linstar des autres détenus detenteurs de fonction etentretiennent des relations amicales, affectives, voire sexuelles, avec d’autres hommes comme en veut l'usage dans les lieux d'enfermement.

Dans la suite du propos, trois cas particuliers seront esquissés : la destinée des fenmmes lesbiennes, des homosexuels juifs et enfin l'incorporé de force des homosexuels dans l'armée allemande.

S'agissant des lesbiennes, il reste très difficile d'etablir combien d'entre elles ont été internées en camp de concentrationenraison de leur orientaons breuses archives ont été détruites. Par ailleurs, seules quelques traces éparses attestent de leur présence en camp puisque dans les registres, la mention
(6) Pieter Koenders, «Die
Bekämpfung der Homose in den besetzten Niederlanden ", in Nationalsozialistischer Terror

(7) Pieter Koenders, «Die in den besetzer Homosexualitä in Nationalsozialistischer Terror
gegen Homosexuelle. Verdrängt

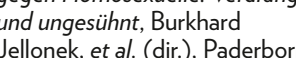
Jellonek, et al. (dir.), Paderborn, 
«lesbienne» figure toujours comme un addendum, c'est-à-dire comm un motif secondaire d'internement comme le rappelle, à juste titre, Claudia Schoppmann dans sa contribution. Concernantles Juifs homosexuels, nou disposons d'informations tout aussi parcellaires à leur sujet ${ }^{8}$. Plusieurs cas toutefois nous permettent de mieux saisir la double peine qui leur était infligée. À titre d'exemple Hans Meyersohn déporté au camp de Sachsenhausen près de Berlin est condamné en 1937 par la justice nazie en tant que personne de «race juive» pour avoir eu des relation sexuelles avec des hommes aryens'. Lo de son procès, il écope de deux chef d'inculpation : le premier pour homosexualité et le second pour opprobre racial contre le peuple allemand. L prononcé de son jugement, daté du 3 février 1937, stipule que le «caractère juif de l'homosexuel Hans Meyersoh constitue un facteur aggravant. Suite à cela, il est interne à Sachsenhausen. Et comme tous les homosexuels juifs, est placé en quarantaine dans le Block réservé aux homosexuels - et non avec les autres Juifs qui sont internes dans «petit camp». Ce cas, ainsi que la destinée des autres hommes répondant aux deux mêmes stigmates, sont particulièrement interessants en raison de lexception que font les nazis. Dès lor qu'il s'agit d'homosexuels, que ceuxci soient «aryens » ou «juifs», ils sont regroupés dans les mêmes baraques et cela afin deviter la "propagation de l'homosexualité au reste du camp. Toutefois, dans certaines condition précises, les homosexuels peuvent être libérés, notamment, lorsque l'Allemagne s'engage dans une guerre total pour laquelle chaque homme compte.

Le cas de Heinz F. est assez parlant en la matière dans la mesure où ce n'est qu’après avoir fait l'expérience de plusieurs camps qu'il est incorpore dans la Wehrmacht. Sa biographie rédigée pa Rainer Hoffschildt dans un ouvrage su es homosexuels de Hanovre mérite d'être esquissée en quelques lignes ${ }^{10}$. Né en 1905, Heinz F. entame des études de droit apres son baccalauréat. Il s'installe par la suite à Munich où il développe un cercle de sociabilité homosexuel. Le 17 décembre 1935, il est arrêté pa la Gestapo. Son arrestation fait suite comme celle d'une vingtaine d'autres homosexuels presumés, à l'arrestation d'une de ses connaissances. Il est d'abord interné à la prison de Bielefeld, ensuite il est transfere à la prison de Munich et enfin a Dachau durant deux ans. En juillet 1937, il est libere. Un an plus tard, il est à nouveau arrêté et incarcéré. La raison de sa seconde arrestation reste méconnue. Cette fois, il est interné Buchenwald et y reste jusqu'en 1942 Affectè à la compagnie pénitentiaire Heinz F. dit avoir de la chance, puisqu'il est au concassage. Avec un autre homosexuel de Berlin, ils remplissent de wagonnets de pierres issues de la carrière. En 1942, il est ensuite transféré a Natzweiler. Cette période est selon ses dires la pire de toutes. A la fin de l'année 1942, il est de nouveau transéré, à Sachsenhausen. Ace moment, les industries qui exploitent les concentrationnaires ont besoin de main-d'ouvre supplémentaire. Pour cette raison, le commando disciplinaire lui est èpargné et il est affecte aux ateliers de la firme aeronautique Heinkel. Fin 1944, la Gestapo ordonne sa liberration de camp et son transfert a la prison de Munich. Le $1^{\text {er }}$ mai 1945, il est incorporé de force dans la Wehrmacht. Son cas n'est pa unique puisque rien quâ Sachsenhausen 357 autres internés sont libéré fin 1944 dans le but dêtre inmédiatement envoyés sur le front (Calic, 2012).
Résistance, libertés

et législations progiressives à l'égard des

homosexuels

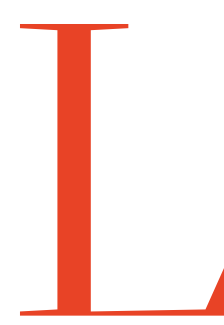

a période de la Second Guerre mondiale ne constitue pas unquement une époque de persecution pour les homosexuels, elle constitue aussi un moment fort d'engagemen contre le nazisme et nombre de femme et hommes homosexuels se sont distingués en ce sens. Par ailleurs, et ce point sera développe dans un second temps, la Seconde Guerre mondiale constitue aussi une periode durant laquelle certains Ėtats européens ont engagé de réformes progressistes en faveur des homosexuel-le-s.

De nombreux hommes et femme bisexuel-le-s et homosexuel-le-s se son en effet engages dans des mouvement de resistance. Il en va ainsi en France, par exemple de Daniel Cordier, qui fut secrétaire de Jean Moulin en 1942-1943. Demême concernant Pascal Copeau,qu fut l'un des fondateurs du Conseil natio- nal de la Résistance (Leenhardt, 1994) ou l'artiste lesbienne Claude Cahun qui participa activement, avec sa compagne, aux mouvements de résistance sur lîle de Jersey, jusquà son arrestation par la Gestapo en 1944. Avec l'historien Michael Sibalis nous pouvons même proposer, comme hypothèse de travail, que les homosexuels etaient des résistants idéaux, car ayant «Ihabitude de la pratique de la clandestinité» et généralement sans conjoints ni enfants, ils pouvaient plus facilement «faire ce saut dans le néant qui est la résistance ${ }^{11}$.»

Toute une partie de l'histoire des homosexuel-le-s dans les mouvements de résistance reste à écrire. Seuls quelques eléments fragmentaires nous sont jusqu’a présent parvenus. Ainsi, l'historien tcheque Jan Seidl a lui aussi retrouvé la trace d'homosexuels et de lesbiennes tchèques qui ont participé aux réseaux de résistance. En premier leu, il sagit d'un reseau constitue autour d'un prêtre, Otakar Zadražil (1900-1945), religieux du monastère des Augustins à Brno. En second lieu, un couple de femmes s'est distingué à Prague en constituant un réseau dont tous les membres (hommes et femmes) étaient homosexuels. Les sources, fragmentaires, attestent que ce groupe a été arrêté et déporté en tant que prisonniers politiques (Seidl, 2014, p. 263-64). De même, Florence Tamagne, auteure d'une remarquable contribution dans ce dossier, a pu montrer dans de précédentes recherches que même dans les camps de concentration, des homosexuels se sont distingués au sein des réseaux de résistance intérieure. Robert Oelbermann, un des leaders des mouvements de jeunesse interdits par les nazis qui fut accusé de crime contre le \$175 et condamné en septembre 1936 à 21 mois de travaux forcés compte parmi
(10) Rainer Hoffschildt, «Wegen Jahre in Konzentrationslagern das Bericht eines Zeitzeugen ",
in Rainer Hoffschildt, Olivia. Die Tabus Homosexualititit und der Verfolgung der Homosexuelle
in Hannover, Hannover,
Sellstverlag. 1992, p. 130-33.

(11) Julian Jackson "Lhomosexualité au XXe siècle climats, problèmes, La Ligne
d'ombre, ${ }^{\circ} 2$ mai 2007 .

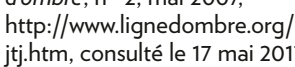


ceux-ci ${ }^{12}$. «Il organisa un groupe de résistance qui parvintà fédérer déporté homosexuels et politiques à Sachsenhausen. [...] Apres la guerre, il fut l'u des rares déportés homosexuels à recevoir le titre de "victime de guerre" en vertu de ses engagements politiques ${ }^{13}$. Guerre mondiale constitue aussi un moment propice à l'établissement de législations plus favorables aux homosexuels. Quelques Etats europeens s'engagent dans un processus de dépénalisation des relations homosexuelles (qui aligne ainsi sa législation sur celle du Danemark qui avait dépenalisé le relations homosexuelles en 1933). En Suisse, l'introduction d'un nouveau Code pénal fédéral en 1942 particip de cette même dynamique de modernisation du droit de la sexualité. Dan ce pays, les cantons alémaniques avaien jusqualors tendance à reprimer l'homosexualité sur le principe du \& 175 allemand tandis que les cantons latin reprenaient majoritairement le droit français ou italien qui ne condamnaient pas l'homosexualite. Enfin, le dernie Etat européen às 'engager dans la voie de la dépénalisation est la Suède, en 1944 Largument invoqué par les tenants de la réforme avait pour objectif de limiter la marge de manouvre des maitre chanteurs qui s'en prenaient de manière privilegiee aux homosexuels. Ce mouvement de dépenalisation progressive et de reconnaissance des formes de vie homosexuelles, impulsé prioritarement par les Etats scandinaves e neutres, c'est ensuite progressivement étendu a toute l'Europe après-guerre, comme l'Histoire l'a montré.

'histoire dont il est question, cell des homosexuel-le-s d'Europe duran
À un autre niveau, la Second à commencer par l'Islande en 1940

la Seconde Guerre mondiale est loin d’avoir été écrite, de nombreuses piste de recherches restent à être explorée tout comme d'autres affinées, notamment grâce aux travaux de jeunes chercheurs dont nous saluons l'audace. Ce numéro spécial de la revue Témoigner n’est pas en reste puisqu'il offre à la foi des perspectives approfondies de chercheuses etchercheurs reconnus dans le domaine de l'Histoire du nazisme et de laDéportation, mais aussi les premières synthèses de travaux très récents de jeunes chercheurs dont gner a la primeur de publier les résulats.

Régis Schlagdenhauffen ant la Seconde G mondiale ", Revue d'ethique et
théologie morale, n' (13) Andreas Sternweiler
Homosexuelle aus der Jugendbewegung ", in Joachir
Müller \& Andreas Sternweiler, Somosexuelle Männer im KZ Sachsenhausen, Berlin, Schwul s
Museum Berlin, Rosa Winkel. 2000.
BIBLIOGRAPHIE

- Bertrand, Mickael (2011) : La Déportation pour motif d'homosexualité en

- Calic, Edouard (2012) : Himmler et l'empire SS, Paris, Nouveau Monde - Gruu, Günter (2004): Homosexualität in der NS-Zeit. Dokumente einer - Heger, Heinz (1972) : Die Männer mit dem rosa Winkel. Der Bericht eines Hoffschite Painer (1992) - Hoffschildt, Rainer: (1992) «Wegen Homosexualität beinahe zehn Jahre in Konzentrationslagern - das Bericht eines Leitzeugen n' in
Rainer Hoffschildt, Olivia. Die bisher geheime Geschichte des Tabus Homosexualitiät und der Verfolgung
Hannover, Selbstverlag, p. 130-33.

- Jellonek, Burhard (1990): Homosexuelle unter dem Hakenkreuz. Die - Kirchknopf, Johann Karl (2017): : «'Anschluss, une annexion sexuelle?»,
in Régis Schlagdenhauffen et al. (dir.), Homosexuels en Europe. - Koenders, Pieter (1996): Tussen christelijk réveil en seksuele revolutie. Bestrijiding van zededloosheid, met de nadruk op repressie van Koonders, Pieter (2002) : « Die Bekämpfung der Homosexualität in
den besetzten Niederlanden », in Nationalsozialistischer Terror gegen Homosexuelle. Verdrangt und ungesühinnt, Paderborn, Ferdinand Schoningh, p. 263-72

- Leenhardt, Pierre (1994): Pascal Copeau : 1908-1982 : I'histoire préfêre

- Mayer, Angela (2002): : "Schwachsinn höheren Grades". Zur ungesühnt, Burkhard Jellonnek, et al. (diri). Paderborn, Schöningh p. 83-96.

- Mufsmann, Olaf (dir.) (2000)
Bad Münstereifel, Westkreuz

- Rahe, Thomas (1999) (dir.), Beiträge zur Geschichte der nationalsozialistischen Verfolgung in Norddeutschland. Verfolgung von - Rozec, Thomas (2011) : Le ll/e Reich et les homosexuels, Paris, Hermann. - Schlagdenhauffen, Régis (2011): Triangle rose, Paris, Autrement.

- Schlagdenhauffen, Régis (2011) : « "Le Dodenherdenking à
|'Homononument d'Amsterdam 》, Témoigner. Entre Histoire et Mémoire, $\mathrm{n}^{\circ} 110$, p. 114-126.

- Schlagdenhauffen, Régis (dir.) (2017): Homosexuel-le-s en Europe
pendant la Seconde Guerre mondiale, Paris, Nouveau Monde.

- Schlegel, Willhart (1967) : Das Großse Tabu. Zeugnisse und Dokumente

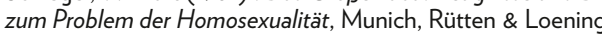

- Schoppmann, Claudia (1997): Nationalsozialistische Sexualpolititi und
weibliche Homosexualititt, Pfaffenweiler Centaurus

- Schwab, Jean-Luc (2010) : Rudolf Brazda, Itinéraire d'un triangle rose,
- Seel, Pierre et Le Bitoux, Jean (1994) : Moi Pierre Seel déporté - Seidl, Jan et al (2014) : Tepla Praha. Pruvodce po queer historii hlavniho 